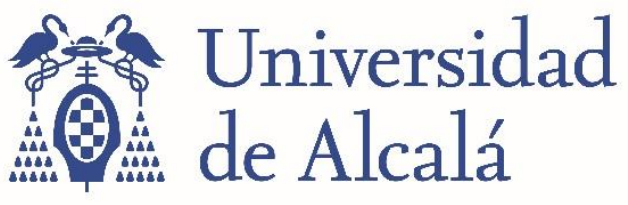

BIBLIOTECA

Document downloaded from the institutional repository of the University of Alcala: http://ebuah.uah.es/dspace/

This is a postprint version of the following published document:

Gómez García, R., Yang, L. \& Muñoz Ferreras, J.M. 2020, "Balanced quasielliptic-type combline diplexer with multiextracted-pole junction/output sections", IEEE Microwave and Wireless Components Letters, vol. 30, no. 6, pp. $569-572$

Available at http://dx.doi.org/10.1109/LMWC.2020.2990084

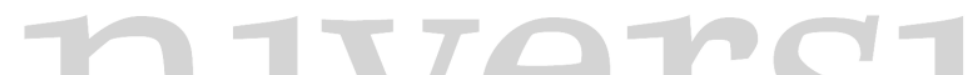

(C) 2020 IEEE. Personal use of this material is permitted. Permission from IEEE must be obtained for all other users, including reprinting/republishing this material for advertising or promotional purposes, creating new collective works for resale or redistribution to servers or lists, or reuse of any copyrighted components of this work in other works.

(Article begins on next page)

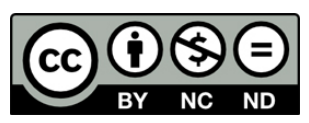

This work is licensed under a

Creative Commons Attribution-NonCommercial-NoDerivatives

4.0 International License. 


\title{
Balanced Quasi-Elliptic-Type Combline Diplexer With Multi-Extracted-Pole Junction/Output Sections
}

\author{
Roberto Gómez-García, Senior Member, IEEE, Li Yang, Member, IEEE, \\ and José-María Muñoz-Ferreras, Member, IEEE
}

\begin{abstract}
A class of coupled-multi-line quasi-elliptic-type balanced diplexer device with closely-spaced channels and its balanced coupling-routing-diagram formalism to simultaneously model its differential- and common-mode operation are reported. It employs third-order combline-type bandpass filters (BPFs) in its filtering channels, whose resonating lines are connected at one of their extremes to the symmetry plane of the balanced-circuit structure (i.e., virtual grounds for the differential mode), along with a multi-extracted-pole/coupled-multi-line dual-band BPF junction and single-band BPF output cells. In differential-mode operation, the dual-band BPF junction adds one in-band pole to each diplexer channel and three transmission zeros (TZs) to both channels, whereas the single-band BPF output cells incorporate one in-band pole and two TZs in their corresponding channels. In this manner, increased-selectivity differential-mode BPF transfer functions for the diplexer channels when compared to those of its third-order combline-type BPFs are obtained. Grounded resistors are connected at the symmetry plane of the balanced diplexer for all the resonating lines of the combline-type BPFs, which become detuned with regard to the poles associated to the dualband BPF junction and the single-band BPF output cells under common-mode excitation. This allows for high common-mode suppression levels in wide spectral ranges to be realized for both channels while maintaining the same TZs as in differentialmode operation. For practical-validation purposes, a $1.6 / 1.8-\mathrm{GHz}$ microstrip prototype is manufactured and tested.
\end{abstract}

Index Terms-Balanced diplexer, balanced filter, commonmode suppression, differential-mode diplexer, differential-mode filter, microstrip diplexer, planar diplexer, transmission zero (TZ).

\section{INTRODUCTION}

In the last few years, the design of balanced/differentialmode microwave passive circuits has attracted considerable attention due to their higher immunity to common-mode noise and electromagnetic-interference phenomena. In particular, special emphasis has been done in the development of planar bandpass filters (BPFs) with differential-mode operation, such as those reported in [1]-[8]. These balanced BPFs are based on distinct operational mechanisms, going from mirrored or duplicated coupled-resonator to signal-interference circuit networks for single- and multi-band applications, which feature both static and spectrally-adaptive differential-mode filtering transfer functions in terms of center frequency and bandwidth.

Regarding differential-mode planar multiplexers for modern balanced multi-channel/full-duplex transceivers, less efforts

Manuscript received February 24, 2020; accepted April 20, 2020. This work was supported by the Spanish Ministry of Economy, Industry, and Competitiveness (State Research Agency) under Project TEC2017-82398-R and by the GOT ENERGY TALENT (GET) fellowship programme co-funded by the EU as part of the H2020-MSCA-COFUND programme (Grant Agreement number 754382). (Corresponding author: Roberto Gómez-García.)

R. Gómez-García, L. Yang, and J.-M. Muñoz-Ferreras are with the Department of Signal Theory and Communications, University of Alcalá, Polytechnic School, Alcalá de Henares 28871, Madrid, Spain (e-mails: roberto.gomez.garcia@ieee.org; li.yang@uah.es; jm.munoz@uah.es).

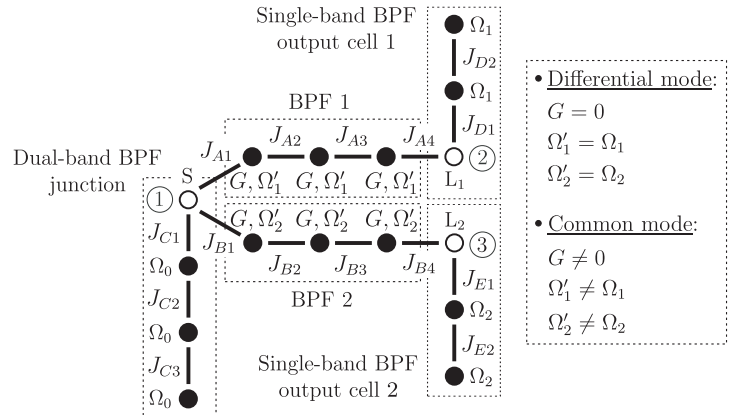

Fig. 1. Normalized coupling-routing diagram of the proposed balanced diplexer (black circles: resonating nodes tuned at $\Omega_{0}, \Omega_{1}, \Omega_{2}, \Omega_{1}^{\prime}$, and $\Omega_{2}^{\prime}$; white circles: unitary source $(\mathrm{S})$ and unitary loads $\left(\mathrm{L}_{1}\right.$ and $\left.\mathrm{L}_{2}\right)$; continuous lines: couplings; $J$ variables: normalized admittance-inversion constants; $G$ : normalized conductance).

have been done in their conception. Some examples to be highlighted are those in [9]-[14], which mostly consist of designs with largely-frequency-spaced channels so that isolation is not critical. In [15] and [16], balanced single- and dual-band diplexers with frequency-contiguous channels were presented, respectively. However, they do not show transmission zeros (TZs) at both passband sides and, in [16], high common-mode suppression is limited to the differential-mode in-band regions.

In this letter, inspired in the single-ended diplexer concept in [17], a quasi-elliptic-type balanced diplexer with multiple TZs for differential- and common-mode operation along with its balanced coupling-routing-diagram formalism are presented in Section II. It employs a multi-extracted-pole dual-band BPF junction and multi-extracted-pole single-band BPF output cells. They enable to increase the order of the diplexer channels with regard to the ones of its embedded low-order BPFs and their selectivity via multi-TZ creation. In Section III, a microstrip implementation of this balanced diplexer that uses coupled-multi-line sections as in [17] for the junction and the output cells, along with embedded combline-type third-order BPFs, is built and tested for practical-validation purposes.

\section{Theoretical Foundations}

The normalized coupling-routing diagram associated to the proposed balanced diplexer architecture is depicted in Fig. 1. As shown, in this particular realization, it is composed of the following parts: (i) in-line third-order BPFs-BPFs 1 and 2 for the lower and upper channels, respectively-, (ii) a dual-band BPF junction, and (iii) single-band BPF output cells-cells 1 and 2 for the lower and upper channels, respectively. Nevertheless, this concept is scalable to higher-order multiplexers with more-than-two channels by increasing the order of the in-line BPFs and the number of bands of the junction accordingly.

For the differential-mode operation, the roles of the different constituent parts of the balanced diplexer are described below: 

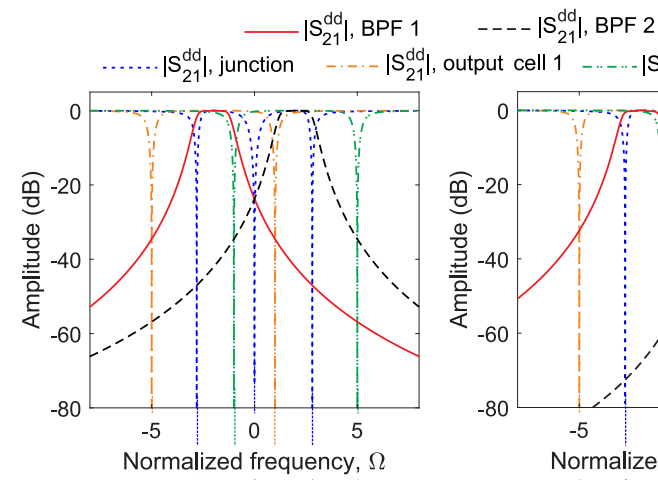

$$
{ }_{21}^{\text {dd }} \mid \text {, output cell } 1 \ldots-\cdots\left|S_{21}^{\text {dd }}\right| \text {, output cell } 2
$$

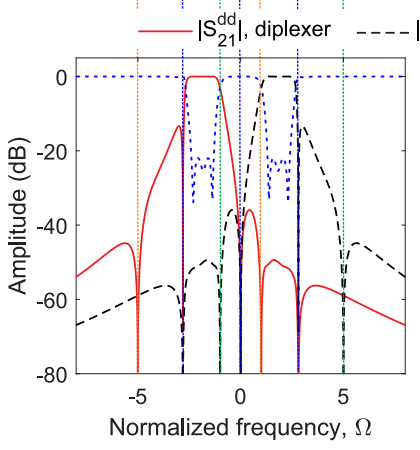

(a)
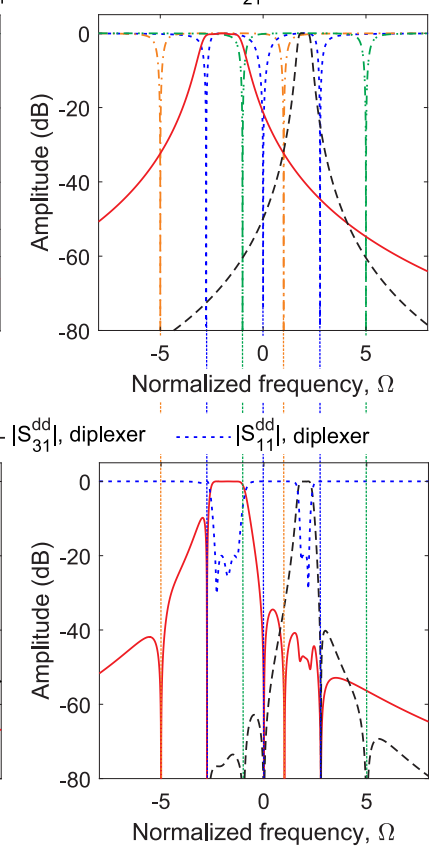

(b)

Fig. 2. Theoretical examples of frequency-symmetrical and frequencyasymmetrical power-transmission $\left(\left|S_{21}^{\mathrm{dd}}\right|\right.$ and $\left.\left|S_{31}^{\mathrm{dd}}\right|\right)$ and input-reflection $\left(\left|S_{11}^{\mathrm{dd}}\right|\right)$ responses of the coupling-routing diagram in Fig. 1 associated to the proposed balanced diplexer in differential-mode operation and $\left|S_{21}^{\mathrm{dd}}\right|$ responses of its constituent low-order filters (BPFs 1 and 2 for the lower and upper channels, respectively), dual-band junction, and single-band output cells (output cells 1 and 2 for the lower and upper channels, respectively). (a) Frequency-symmetrical example. (b) Frequency-asymmetrical example.

- The in-line third-order BPFs provide a lower-order approximation without finite TZs to the intended transfer functions of the overall channel diplexers. In this manner, these BPFs are designed as follows: (i) the normalized natural frequencies of their resonating nodes for the lower and upper channels-i.e., $\Omega_{1}^{\prime}$ and $\Omega_{2}^{\prime}$, respectively-are selected to be equal to their desired normalized center frequencies-i.e., $\Omega_{1}$ and $\Omega_{2}$, respectively—and (ii) their 3-dB normalized bandwidths are synthesized to be nearly equal to those required for the overall diplexer channels.

- The dual-band BPF junction, whose resonating nodes are tuned at $\Omega_{0}$, exhibits a dual-band transfer function with one-pole passbands-as well as the poles at $\pm \infty$ - and one $\mathrm{TZ}$ at each passband side-i.e., three TZs in total. The lower and upper finite poles at $\Omega_{p 1}$ and $\Omega_{p 2}$ are transferred to the lower and upper channels by imposing them to be equal to $\Omega_{1}$ and $\Omega_{2}$, respectively—so that their orders are increased by one-, whereas the three TZs at the normalized frequencies $\Omega_{z 1}, \Omega_{z 2}$, and $\Omega_{z 3}$ are shared by both channels. The design formulas associated to the dual-band BPF junction are as follows [17]:

$$
\begin{array}{cc}
\text { TZs: } & \Omega_{z 1, z 3}=\Omega_{0} \mp \sqrt{J_{C 2}^{2}+J_{C 3}^{2}} \quad \Omega_{z 2}=\Omega_{0} . \\
\underline{\text { Poles: }}: \quad \Omega_{p 1, p 2}=\Omega_{0} \mp\left|J_{C 3}\right|=\Omega_{1,2} .
\end{array}
$$

- The single-band BPF output cells have a bandpass response with a one-pole transmission band-as well as the poles at $\pm \infty$ - and one $\mathrm{TZ}$ at each passband side- i.e., two TZs in total. Each of these output cells transfers its finite pole $-\Omega_{p 3}$ for the cell 1 and $\Omega_{p 4}$ for the cell 2-and two TZs- $\Omega_{z 4}$ and $\Omega_{z 5}$ for the cell 1 and $\Omega_{z 6}$ and $\Omega_{z 7}$ for the cell 2-to the channel at which they are inserted. Their synthesis equations are provided below:

$$
\begin{aligned}
& \underline{\text { TZs: }}: \quad \Omega_{z 4, z 5}=\Omega_{1} \mp\left|J_{D 2}\right| \quad \Omega_{z 6, z 7}=\Omega_{2} \mp\left|J_{E 2}\right| \text {. } \\
& \text { Poles: } \quad \Omega_{p 3}=\Omega_{1} \quad \Omega_{p 4}=\Omega_{2} \text {. }
\end{aligned}
$$

As a result, by means of the action of the dual-band BPF junction and the single-band BPF output cells, the order of the diplexer channels and their selectivity are increased through multi-TZ generation with regard to those offered by their constituent in-line third-order BPFs. This allows for quasi-elliptictype sharp-rejection filtering capabilities to be obtained for the overall diplexer device under differential-mode excitation. For illustration purposes, the differential-mode power transmission and reflection responses of theoretically-synthesized frequency-symmetrical and frequency-asymmetrical examples with the coupling-routing diagram in Fig. 1-along with the differential-mode transfer functions of its building parts-are represented in Fig. 2. These design examples, whose parameter values are listed in Table I, were obtained by means of the design formulas (1)-(4) and fine optimization with Matlab. As proven, the filtering selectivity of the diplexer channels is remarkably enhanced when compared to that offered by the inline third-order BPFs by means of in-band-pole augmentation and the creation of five different TZs in each diplexer channel.

For the common-mode operation, the resonating nodes of the in-line third-order BPFs become detuned with regard to the poles created by the dual-band BPF junction and singleband BPF output cells and they are assumed to be lossy (i.e., $G \neq 0$ in Fig. 1). Consequently, high common-mode suppression levels for each duplexer channel in very-broad bandwidths can be realized. This is illustrated in Fig. 3 for the frequency-symmetrical example in Fig. 2(a), which shows how the differential-mode TZs are maintained under commonmode excitation. Furthermore, as can be seen in Fig. 3, higher values of the normalized-conductance parameter $G$ associated to the detuned lossy resonating nodes in the in-line third-order BPFs lead to increased common-mode suppression levels.

TABLE I

Design Parameters for the Design Examples in Fig. 2

\begin{tabular}{c|cc}
\hline \hline Symmetrical Example & \multicolumn{2}{|c}{ Asymmetrical Example } \\
\hline$J_{A 1}=J_{B 1}=0.7504$ & $J_{A 1}=0.7613$ & $J_{A 2}=0.581$ \\
$J_{A 2}=J_{B 2}=0.5398$ & $J_{A 3}=0.7678$ & $J_{A 4}=0.9165$ \\
$J_{A 3}=J_{B 3}=0.6694$ & $J_{B 1}=0.4643$ & $J_{B 2}=0.2057$ \\
$J_{A 4}=J_{B 4}=0.905$ & $J_{B 3}=0.2367$ & $J_{B 4}=0.5656$ \\
$J_{C 1}=1.3267 \quad J_{C 2}=1.974$ & $J_{C 1}=1.3595$ & $J_{C 2}=1.9163$ \\
$J_{C 3}=2 \quad J_{D 1}=J_{E 1}=1.1035$ & $J_{C 3}=2$ & $J_{D 1}=J_{E 1}=1.0145$ \\
$J_{D 2}=J_{E 2}=3 \quad \Omega_{0}=0$ & $J_{D 2}=J_{E 2}=3 \quad \Omega_{0}=0$ \\
$\Omega_{1}=\Omega_{1}^{\prime}=-2 \quad \Omega_{2}=\Omega_{2}^{\prime}=2$ & $\Omega_{1}=\Omega_{1}^{\prime}=-2 \quad \Omega_{2}=\Omega_{2}^{\prime}=2$ \\
$G=0$ & \multicolumn{2}{|c}{$G=0$} \\
\hline \hline
\end{tabular}

\section{EXPERIMENTAL RESULTS}

For practical-validation purposes, a microstrip prototype that implements the balanced diplexer concept suggested in Fig. 1 has been manufactured and tested. Its lower and upper closelyspaced channels were designed to exhibit center frequencies of $1.6 \mathrm{GHz}$ and $1.8 \mathrm{GHz}$ and $3-\mathrm{dB}$ absolute bandwidths 


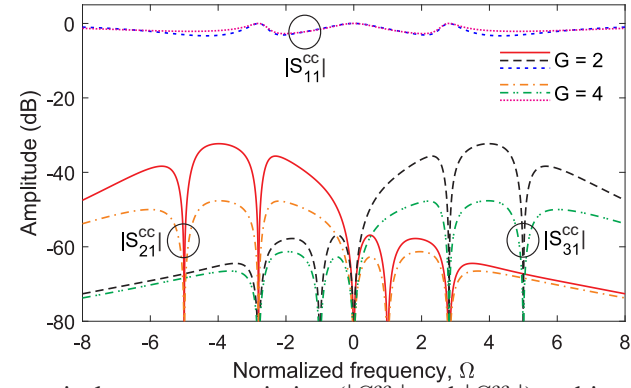

Fig. 3. Theoretical power transmission $\left(\left|S_{21}^{\text {cc }}\right|\right.$ and $\left.\left|S_{31}^{\text {cc }}\right|\right)$ and input-reflection $\left(\left|S_{11}^{\mathrm{cc}}\right|\right)$ responses of the coupling-routing diagram in Fig. 1 associated to the proposed balanced diplexer in common-mode operation for the symmetrical example in Fig. 2(a) $\left(\Omega_{1}^{\prime}=-4, \Omega_{2}^{\prime}=4, G=2\right.$ and 4 , and the remaining design parameters as indicated in Table I).

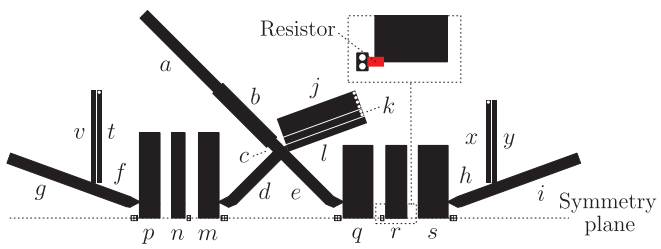

Fig. 4. Layout of the manufactured microstrip prototype of balanced diplexer (microstrip substrate Rogers 4003C: relative dielectric permittivity $\varepsilon_{r}=3.38$ ( \pm 0.05 ), dielectric thickness $H=1.524 \mathrm{~mm}$, metal thickness $t=17.8 \mu \mathrm{m}$, and dielectric loss tangent $\tan \delta_{D}=0.0027$; resistors: $15-\Omega$ resistance from Panasonic; ground connections: 1/0.6-mm-diameter via holes; dimensions in $\mathrm{mm}-w$ stands for width, $l$ for length, and $s$ for inter-line spacing of straight microstrip-line segments without corner regions: $w_{a}=w_{c}=w_{d}=$ $w_{e}=w_{f}=w_{g}=w_{h}=w_{i}=3.38, w_{b}=4.5, w_{j}=6, w_{k}=w_{l}=2.2$, $w_{m}=w_{p}=6.5, w_{n}=4.18, w_{q}=w_{s}=9.4, w_{r}=6.6, w_{v}=w_{t}=$ $w_{x}=w_{y}=1.5, l_{a}=33, l_{b}=24, l_{c}=2.5, l_{d}=l_{e}=20, l_{f}=l_{h}=$ $11.5, l_{g}=l_{i}=27, l_{j}=l_{k}=l_{l}=26.3, l_{m}=l_{n}=l_{p}=26.9$, $l_{q}=l_{r}=l_{s}=22.9, l_{v}=l_{t}=29, l_{x}=l_{y}=25.9, s_{j k}=s_{k l}=0.3$, $s_{m n}=s_{n p}=4.21, s_{q r}=s_{r s}=3.65$, and $\left.s_{v t}=s_{x y}=0.3\right)$.

equal to $120 \mathrm{MHz}$-i.e., $7.5 \%$ and $6.7 \%$ in relative terms. The layout of the developed microstrip balanced diplexer is shown in Fig. 4. As can be seen, third-order combline-type BPFs are used in the diplexer channels. The multi-extractedpole dual-band BPF junction and single-band output cells are realized with coupled-multi-line stages which are made up of in-parallel-inter-coupled quarter-wavelength resonating lines ended in open- and short-circuits at their two extremes. The reminder admittance inverters are designed as quarterwavelength transmission-line segments. Besides, grounded resistors are connected in the three resonating lines of the combline-type BPFs at the symmetry plane of the balanced diplexer. In this manner, the operational principle of the balanced diplexer concept in Section II is realized as follows:

- In differential-mode operation, the resonating lines of the combline-type BPFs are terminated in virtual grounds at the symmetry plane, so that their natural frequencies are located at the spectral positions of the in-band poles of the coupled-multi-line dual-band BPF junction and singleband BPF output cells in each diplexer channel.

- In common-mode operation, the resonating lines of the combline-type BPFs are ended in grounded resistors at the symmetry plane, so that they become lossy resonators that are detuned with regard to the locations of the inband poles associated to the coupled-multi-line dual-band BPF junction and single-band BPF output cells.

The simulated and measured power transmission and inputreflection parameters of the constructed microstrip prototype

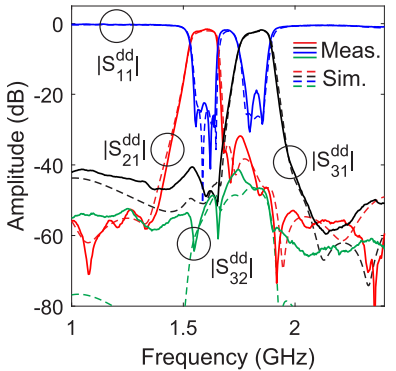

(a)

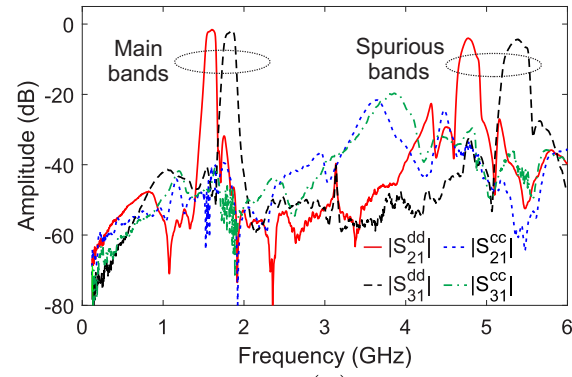

(c)

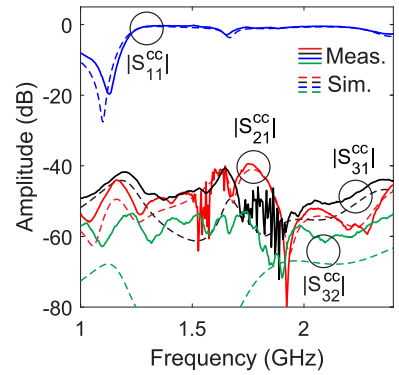

(b)

(d)

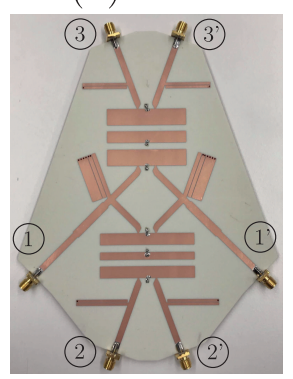

Fig. 5. Simulated (Ansys HFSS) and measured power transmission (differential mode: $\left|S_{21}^{\mathrm{dd}}\right|$ and $\left|S_{31}^{\mathrm{dd}}\right|$; common mode: $\left|S_{21}^{\mathrm{cc}}\right|$ and $\left.\left|S_{31}^{\mathrm{cc}}\right|\right)$, output-isolation (differential mode: $\left|S_{32}^{\mathrm{dd}}\right|$; common mode: $\left|S_{32}^{\mathrm{cc}}\right|$ ), and input-reflection (differential mode: $\left|S_{11}^{\text {dd }}\right|$; common mode: $\left.\left|S_{11}^{\text {cc }}\right|\right)$ parameters of the manufactured microstrip prototype of balanced diplexer and photograph. (a) $\left|S_{21}^{\mathrm{dd}}\right|,\left|S_{31}^{\mathrm{dd}}\right|$, $\left|S_{32}^{\text {dd }}\right|$, and $\left|S_{11}^{\text {dd }}\right|$. (b) $\left|S_{21}^{\text {cc }}\right|,\left|S_{31}^{\text {cc }}\right|,\left|S_{32}^{\text {cc }}\right|$, and $\left|S_{11}^{\text {cc }}\right|$. (c) $\left|S_{21}^{\text {dd }}\right|,\left|S_{31}^{\text {dd }}\right|,\left|S_{21}^{\text {cc }}\right|$, and $\left|S_{31}^{\mathrm{cc}}\right|$ (measurements in a broader frequency interval). (d) Photograph.

of balanced diplexer for the differential and common modes are depicted in Fig. 5(a) and (b), respectively, being in fairlyclose agreement. Its measured differential- and common-mode power transmission parameters for a broader frequency interval are represented in Fig. 5(c) and its photograph in Fig. 5(d). The main measured characteristics of the measured balanced diplexer for the lower and upper channels under differentialmode excitation are as follows: center frequencies of $1.59 \mathrm{GHz}$ and $1.82 \mathrm{GHz}, 3-\mathrm{dB}$ absolute bandwidths equal to $116 \mathrm{MHz}$ and $127 \mathrm{MHz}$-i.e., equal to $7.3 \%$ and $7 \%$ in relative terms-, minimum in-band power-insertion-loss levels of $1.6 \mathrm{~dB}$ and $1.8 \mathrm{~dB}$, minimum in-band input-power-matching levels of 22.4 $\mathrm{dB}$ and $18.9 \mathrm{~dB}$, minimum in-band output-isolation levels of $40.1 \mathrm{~dB}$ and $48.7 \mathrm{~dB}$, and 30-dB-attenuation-referred upper stopbands in the ranges $1.67-4.21 \mathrm{GHz}$ and $1.95-5.12 \mathrm{GHz}$. The measured common-mode suppression levels are higher than $40.1 \mathrm{~dB}$ and $46 \mathrm{~dB}$ in the $3-\mathrm{dB}$ passbands and above $19.7 \mathrm{~dB}$ throughout the $0-6-\mathrm{GHz}$ band.

\section{CONCLUSion}

A type of multi-extracted-pole balanced diplexer with sharprejection differential-mode filtering responses for its channels through multi-TZ generation and its balanced couplingrouting-diagram formulation have been reported. The inclusion of grounded resistors in the symmetry plane of the balanced diplexer, along with the referred TZs, enable high commonmode suppression levels to be attained for both channels in broad spectral ranges. For experimental-verification purposes, a 1.6/1.8-GHz microstrip prototype that employs third-order combline-type BPFs in its closely-spaced channels, along with a coupled-multi-line dual-band BPF junction and single-band BPF output cells, has been developed and characterized. 


\section{REFERENCES}

[1] W. J. Feng, W. Q. Che, and Q. Xue, "The proper balance: Overview of microstrip wideband balanced circuits with wideband common mode suppression," IEEE Microw. Mag., vol. 16, no. 5, pp. 55-68, Jun. 2015.

[2] F. Martin, L. Zhu, J. Hong, and F. Medina, Balanced Microwave Filters, 1st ed. New York, NY, USA: Wiley-IEEE Press, 2018.

[3] R. Gómez-García, R. Loeches-Sánchez, D. Psychogiou, and D. Peroulis, "Multi-stub-loaded differential-mode multi-band bandpass planar filters," IEEE Trans. Circuits Syst. II, Exp. Briefs, vol. 65, no. 3, pp. 271-275, Mar. 2018.

[4] R. Gómez-García, J.-M. Muñoz-Ferreras, W. Feng, and D. Psychogiou, "Balanced symmetrical quasi-reflectionless single- and dual-band bandpass planar filters," IEEE Microw. Wireless Compon. Lett., vol. 28, no. 9, pp. 798-800, Sep. 2018.

[5] D. J. Simpson and D. Psychogiou, "Coupling matrix-based design of fully reconfigurable differential/balanced RF filters," IEEE Microw. Wireless Compon. Lett., vol. 28, no. 10, pp. 888-890, Oct. 2018.

[6] M. Fan, K. Song, and Y. Zhu, "Reconfigurable differential filter with constant absolute bandwidth and high common-mode suppression," IEEE Microw. Wireless Compon. Lett., vol. 28, no. 10, pp. 894-896, Oct. 2018.

[7] Q. Liu, J. Wang, G. Zhang, L. Zhu, and W. Wu, "A new design approach for balanced bandpass filters on right-angled isosceles triangular patch resonator," IEEE Microw. Wireless Compon. Lett., vol. 29, no. 1, pp. 5-7, Jan. 2019.

[8] H.-W. Deng, L. Sun, F. Liu, Y.-F. Xue, and T. Xu, "Compact tunable balanced bandpass filter with constant bandwidth based on magnetically coupled resonators," IEEE Microw. Wireless Compon. Lett., vol. 29, no. 4, pp. 264-266, Apr. 2019.

[9] P.-L. Chi and T. Yang, "Novel 1.5-2.4 GHz tunable single-to-balanced diplexer," IEEE Microw. Wireless Compon. Lett., vol. 26, no. 10, pp. 783-785, Oct. 2016.

[10] Y. Zhou, H.-W. Deng, and Y. Zhao, "Compact balanced-to-balanced microstrip diplexer with high isolation and common-mode suppression," IEEE Microw. Wireless Compon. Lett., vol. 27, no. 3, pp. 143-145, Mar. 2017.

[11] X. Guo, L. Zhu, and W. Wu, "Balanced diplexers based on inner-coupled dual-mode structures with intrinsic common-mode suppression," IEEE Access, vol. 5, pp. 26774-26782, 2017.

[12] A. Fernández-Prieto, A. Lujambio, F. Martín, J. Martel, F. Medina, and R. R. Boix, "Compact balanced-to-balanced diplexer based on split-ring resonators balanced bandpass filters," IEEE Microw. Wireless Compon. Lett., vol. 28, no. 3, pp. 218-220, Mar. 2018.

[13] W. Jiang, Y. Huang, T. Wang, Y. Peng, and G. Wang, "Microstrip balanced quad-channel diplexer using dual-open/short-stub loaded resonator," in Proc. 2016 IEEE MTT-S Int. Microw. Symp., San Francisco, CA, USA, May 22-27, 2016, pp. 1-3.

[14] C.-H. Lee, C.-I. G. Hsu, S.-X. Wu, and P.-H. Wen, "Balanced quad-band diplexer with wide common-mode suppression and high differentialmode isolation," IET Microw. Antennas Propag., vol. 10, no. 6, pp. 599603, Jun. 2016.

[15] A. Fernández-Prieto, A. Lujambio, J. Martel, F. Medina, F. Martín, and R. R. Boix, "Balanced-to-balanced microstrip diplexer based on magnetically coupled resonators," IEEE Access, vol. 6, pp. 1853618547, 2018.

[16] R. Gómez-García, J.-M. Muñoz-Ferreras, L. Yang, and D. Psychogiou, "Contiguous-channel dual-band balanced diplexer," IEEE Microw. Wireless Compon. Lett., vol. 29, no. 5, pp. 318-320, May 2019.

[17] R. Gómez-García, L. Yang, J.-M. Muñoz-Ferreras, and D. Psychogiou, "Single/multi-band coupled-multi-line filtering section and its application to RF diplexers, bandpass/ bandstop filters, and filtering couplers," IEEE Trans. Microw. Theory Techn., vol. 67, no. 10, pp. 3959-3972, Oct. 2019. 